FREITAS, A.I.A. e ROSATO, G.R. Estudo ético e científico sobre o uso de métodos alternativos no ensino de práticas cirúrgicas na Medicina Veterinária. PUBVET, Londrina, V. 5, N. 9, Ed. 156, Art. 1053, 2011.

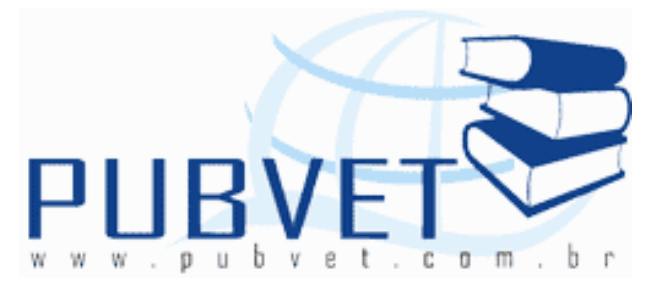

PUBVET, Publicações em Medicina Veterinária e Zootecnia.

\title{
Estudo ético e científico sobre o uso de métodos alternativos no ensino de práticas cirúrgicas na Medicina Veterinária
}

\author{
Andressa Izabel Assis Freitas ${ }^{1}$ e Gustavo Rodrigues Rosato ${ }^{2}$
}

1 Médica Veterinária

2 Aluno da Graduação do curso de Filosofia da Universidade Federal de Uberlândia (UFU)

\section{Resumo}

O trabalho pretende avaliar a eficiência dos métodos alternativos para o ensino de práticas cirúrgicas, fazendo uma comparação com os métodos convencionais de ensino das mesmas, utilizando como exemplo as disciplinas oferecidas pela Faculdade de Medicina Veterinária de Uberlândia (FAMEV/UFU) como métodos convencionais. Como métodos alternativos, foram escolhidos três exemplos: a solução de Larssen modificada, as vídeo-aulas e os programas de softwares com simulações em terceira dimensão, para que seja elaborado um estudo baseado em critérios éticos e científicos, avaliando ambos os métodos. Como critério científico pretende-se constatar as vantagens e desvantagens de tais métodos comparando sua eficiência para concluir a necessidade de seu uso. Como critérios éticos, pretende-se constatar os danos causados aos animais não humanos e a violação de seus interesses que cada método apresenta. Comparando a eficiência e os resultados obtidos nas metodologias de ensino convencionais, com as 
FREITAS, A.I.A. e ROSATO, G.R. Estudo ético e científico sobre o uso de métodos alternativos no ensino de práticas cirúrgicas na Medicina Veterinária. PUBVET, Londrina, V. 5, N. 9, Ed. 156, Art. 1053, 2011.

metodologias que utilizam métodos alternativos, poderá se entender a real necessidade do uso de animais não humanos vivo para fins didáticos.

Palavras-chave: fins didáticos, métodos alternativos, métodos convencionais

\title{
Ethical and scientific study on alternative methods use in the teaching of surgical practice in Medicine Veterinary
}

\begin{abstract}
This project aims to evaluate the alternative method's efficiency for teaching surgical practices, making a comparison with the conventional methods, using as example the subjects offered by Faculdade de Medicina Veterinária de Uberlândia (FAMEV/UFU) as conventional methods, and three examples as alternative methods: Larssen's solution modified, video-classes and 3D simulation's software, in order to make out a study based on ethical and scientific criteria, evaluating both methods. As scientific criterion, seeks to establish the advantages and disadvantages of these methods comparing their efficiency to imply the need to use; as ethical criterion seeks to find the damage to non-human animals and the violation of their interests submitted by each method. Comparing the efficiency and the results obtained in conventional teaching methodologies with the methodologies that use alternative methods, it will be possible to understand the real need for the use of nonhuman animals alive for teaching purposes.
\end{abstract}

Keywords: alternative methods, conventional methods, teaching purposes

\section{Estudo ético baseado no princípio da necessidade}

A vivissecção é a utilização de animais não humanos, que são mantidos em biotérios, para fins didáticos e pesquisas. Mais do que um método científico, o uso de animais se tornou um hábito, e por muito tempo se pensou que sua utilização era extremamente necessária para o avanço da ciência, 
FREITAS, A.I.A. e ROSATO, G.R. Estudo ético e científico sobre o uso de métodos alternativos no ensino de práticas cirúrgicas na Medicina Veterinária. PUBVET, Londrina, V. 5, N. 9, Ed. 156, Art. 1053, 2011.

criando assim um conflito de interesses, onde o bem para os humanos era retirado do sofrimento e sacrifício de outras espécies.

Com a criação de novas tecnologias, foi possível o aperfeiçoamento de métodos alternativos, que procuram principalmente substituir o uso de animais vivos, e podem produzir resultados iguais ou melhores aos obtidos com o uso de modelos convencionais, fazendo possível que ética e ciência andem juntos. No entanto, devido a séculos de maus hábitos, as novas tecnologias de métodos alternativos não conseguiram a aceitação de todos os pesquisadores e educadores.

O princípio ético que será demonstrado, pretende resolver o suposto dilema que é construído, quando se pensa que o ensino de práticas cirúrgicas se faz necessário o uso de animais vivos. Analisando a eficiência dos atuais métodos alternativos no ensino de práticas cirúrgicas, e comparando com os resultados que se pretende alcançar com as metodologias de ensino convencionais, será possível demonstrar que a utilização de animais vivos não se faz mais necessária perante a alternativa do uso de novas tecnologias.

O suposto dilema ético, que envolve a necessidade do uso de animais vivos para que se tenha avanços na ciência e salvar posteriores vidas, é chamado de suposto, pois se trata de um dilema científico e não ético. Quando falamos do valor de uma vida, para o julgamento ético, não há vidas mais valiosas que mereçam ser salvas com o sacrifício de outras. Tirar vidas para salvar vidas, não é uma solução ética, e sim científica. A solução ética deve estudar as conseqüências e necessidades de um ato avaliando por igual os interesses de todos os envolvidos.

As considerações dos interesses devem avaliar tanto os interesses humanos quanto os de outras espécies. Acreditar que os interesses dos humanos valem mais que os interesses de outras espécies, tendo como argumento a superioridade do ser humano é chamado por Peter Singer de especismo. Assim como acreditar que os membros de uma raça são superiores 
FREITAS, A.I.A. e ROSATO, G.R. Estudo ético e científico sobre o uso de métodos alternativos no ensino de práticas cirúrgicas na Medicina Veterinária. PUBVET, Londrina, V. 5, N. 9, Ed. 156, Art. 1053, 2011.

apenas pela razão de pertencerem aquela determinada raça é chamado de racismo. ${ }^{1}$

A demonstração da eficiência dos métodos alternativos e a comprovação da falta de necessidade dos métodos convencionais, que utilizam animais vivos, também não se trata de um argumento ético, pois nem tudo que é desnecessário é antiético. No entanto, o princípio da necessidade, que será defendido, irá demonstrar que além de serem desnecessários cientificamente os métodos convencionais se tornam antiéticos, quando julgados por desrespeitarem o valor da vida e os interesses dos animais.

Apesar dos métodos alternativos ainda apresentarem algumas carências, por não permitirem o treinamento de algumas complicações cirúrgicas, como nos casos de hemorragia e pressão do animal, essas lacunas podem ser preenchidas através de estágios, plantões e residência em um hospital veterinário, ou clínica veterinária, onde 0 aluno terá o acompanhamento de um cirurgião já formado e que poderá instruí-lo de início em procedimentos mais simples, como castrações, até que o aluno esteja preparado para casos mais complicados.

Além de demonstrar que os métodos convencionais não são mais necessário para o ensino de práticas cirúrgicas, o estudo ético deste trabalho também pretende avaliar os danos causados aos animais e demonstrar o real valor de suas vidas que não podem ser substituídas pelo bem de outras espécies, e que o avanço da ciência deve respeitar os limites impostos pelas normas e leis criadas para defender os interesses da parte mais fraca, os animais.

\section{Avaliação da metodologia convencional}

Para entendermos melhor a importância do ensino de práticas cirúrgicas, nos cursos de Medicina Veterinária, será demonstrado quais são os objetivos que se pretende alcançar, e as habilidades que se pretende ensinar

\footnotetext{
${ }^{1}$ SINGER, Peter. Ética Prática. Tradução de Jefferson Luiz Camargo. São Paul: Martins Fontes, 2006. p 65. Tradução de: Practical Ethics.
} 
FREITAS, A.I.A. e ROSATO, G.R. Estudo ético e científico sobre o uso de métodos alternativos no ensino de práticas cirúrgicas na Medicina Veterinária. PUBVET, Londrina, V. 5, N. 9, Ed. 156, Art. 1053, 2011.

aos alunos, assim será possível entender quais são as reais necessidades e benefícios que a formação de Médicos Veterinários cirurgiões, pretende alcançar.

Para a avaliação científica e ética da metodologia convencional de ensino de práticas cirúrgicas foram escolhidas duas disciplinas, sendo a primeira Patologia Clínica Cirúrgica, oferecida ao oitavo período, e a segunda Técnica Operatória e Prática Anestésica, oferecida ao sétimo período, pela Faculdade de Medicina Veterinária da Universidade Federal de Uberlândia (FAMEV/UFU) Uberlândia, MG.

No segundo semestre de 2008, com base em relatos de alunos e pela experiência própria, a disciplina de Patologia Clínica Cirúrgica utiliza-se de cães e principalmente bovinos. Os primeiros foram provenientes do Centro de Controle de Zoonoses de Uberlândia, muitas vezes saudáveis, que eram mantidos vivos no biotério da Faculdade, e os bovinos eram provenientes da fazenda escola Glória, e foram mantidos em um curral no interior da faculdade. O objetivo da disciplina é preparar os alunos para práticas de procedimentos cirúrgicos em animais de pequeno porte e de grande porte. O único método utilizado para reduzir o número de animais é a divisão em grupos de cinco pessoas, no qual cada um será responsável pelas seguintes funções, assepcista, anestesista, um cirurgião e dois auxiliares. O grupo realiza apenas uma vez cada procedimento, não sendo possível o treino da prática cirúrgica de todos os integrantes e em todos os procedimentos, tornando a metodologia ineficaz para formação de um cirurgião. Logo após as cirurgias de treinamento os cães são eutanasiados enquanto os bovinos são mantidos vivos até o término do semestre.

Com o mesmo embasamento citado anteriormente, a disciplina de Técnica Operatória e Prática Anestésica, no primeiro semestre de 2008, utilizou somente cães provenientes do mesmo biotério. Como o objetivo da disciplina é preparar os alunos para a prática de procedimentos cirúrgicos e anestésicos em animais de pequeno porte, que devem ser utilizados ainda vivos com a justificativa de que é necessário preparar os alunos para situações reais onde é 
FREITAS, A.I.A. e ROSATO, G.R. Estudo ético e científico sobre o uso de métodos alternativos no ensino de práticas cirúrgicas na Medicina Veterinária. PUBVET, Londrina, V. 5, N. 9, Ed. 156, Art. 1053, 2011.

possível ocorrer parada cardíaca e respiratória, além de hemorragias. No entanto, ao final dos procedimentos todos os animais são submetidos a eutanásia, isso provoca aos alunos uma desvinculação entre animal e valor de vida, tornando-o apenas um objeto de estudo e as preocupações com a vida do animal que deveriam ser ensinadas tomam-se um caráter negativo.

Analisar os resultados obtidos com tais métodos convencionais, e sua comparação com a necessidade da utilização e sacrifício dos cães, levando em consideração a possibilidade e eficiência dos métodos alternativos, será muito importante para a elaboração de uma conclusão científica e ética que pretende refutar o suposto dilema da necessidade do uso de animais vivos para se ter um ensino de qualidade.

Pode-se observar na metodologia convencional, que o aluno pratica o procedimento uma única vez, sendo que para cada procedimento é utilizado um único animal, o que torna muito grande a quantidade de animais utilizados. Como os animais são sacrificados ao final da aula prática, na maioria dos procedimentos, não é possível que o aluno adquira a prática dos cuidados pósoperatórios e, pelo mesmo motivo, não é possível que se comprove a eficiência do procedimento praticado pelo aluno.

\section{Métodos Alternativos}

Os métodos alternativos são propostas de metodologias didáticas e científicas que pretendem, principalmente, substituir o uso de animais vivos. Para este trabalho, foram escolhidos três diferentes métodos alternativos: a solução de Larssen modificada, as gravações de procedimentos demonstrativos em recursos áudio visuais e os programas de computadores que possibilitam o treinamento virtual de práticas cirúrgicas. É importante lembrar que os métodos alternativos não se limitam aos escolhidos neste trabalho existindo vários outros métodos.

Os métodos alternativos além de serem eticamente recomendáveis para o treinamento de práticas cirúrgicas, também melhoram a qualidade didática dessas práticas, reduzindo suas limitações e possibilitando ao aluno que não 
FREITAS, A.I.A. e ROSATO, G.R. Estudo ético e científico sobre o uso de métodos alternativos no ensino de práticas cirúrgicas na Medicina Veterinária. PUBVET, Londrina, V. 5, N. 9, Ed. 156, Art. 1053, 2011.

concordam com o uso de animais vivos em sala de aula, poderem praticá-la sem sofrer danos morais ou estresse.

\subsection{Solução de Larssen modificada como método alternativo}

O primeiro método alternativo escolhido para o estudo ético do trabalho foi á solução de Larssen modificada, utilizado nas Disciplinas de Técnicas Cirúrgicas e de Ortopedia da Faculdade de Medicina Veterinária e Zootecnia da Universidade de São Paulo (FMVZ/USP), São Paulo, SP.

A solução de Larssen modificada é baseada em uma solução utilizada na Universidade René Descartes, pelo Hospital Cochim, Paris, França. Na solução original foi utilizado $100 \mathrm{ml}$ de formalina a $10 \%, 400 \mathrm{rnl}$ de glicerina líquida, $200 \mathrm{~g}$ de hidrato de cloral, $200 \mathrm{~g}$ de sulfato de sódio, $200 \mathrm{~g}$ de bicarbonato de sódio, $180 \mathrm{~g}$ de cloreto de sódio, $2000 \mathrm{rnl}$ de água destilada. A solução modificada utiliza uma parte da solução original para três partes de água destilada. $^{2}$

O objetivo da utilização da solução de Larssen modificada, como método alternativo para o ensino de práticas cirúrgicas, é a conservação de cadáveres de cães, possibilitando sua utilização para o treinamento das técnicas cirúrgicas, sem que ocorra uma perda na qualidade, mantendo a coloração dos tecidos, a flexibilidade das articulações e evitando o odor emitido pela putrefação. ${ }^{3}$

A utilização da solução de Larssen modificada no ensino de práticas cirúrgicas na FMVZ/USP, em uma análise ética e científica, busca comparar a eficiência do método com os resultados que se pretende alcançar nas disciplinas. A utilização deste método alternativo se mostra muito eficiente por ser capaz de fazer com que os alunos pratiquem várias vezes os procedimentos cirúrgicos, além de se mostrar também conforme as normas e leis que protegem os interesses dos animais ética e juridicamente, pois vários

\footnotetext{
${ }^{2}$ SILVA, R. M. G; MATERA, J. M; MACIEL, A. A. C. Avaliação do método do ensino da técnica cirúrgica utilizando cadáveres quimicamente preservados.

$<$ http://www.hostcentral.com.br/crmv/PDF/v6n1a10.pdf > Acesso em 20. Jul. 2010

${ }^{3}$ Ibidem.
} 
FREITAS, A.I.A. e ROSATO, G.R. Estudo ético e científico sobre o uso de métodos alternativos no ensino de práticas cirúrgicas na Medicina Veterinária. PUBVET, Londrina, V. 5, N. 9, Ed. 156, Art. 1053, 2011.

procedimentos são feitos em um único animal, que já se encontrava morto por outros motivos e conservado pela solução de Larssen modificada, o que reduz o número de animais vivos utilizados.

A "redução" é um dos três princípios éticos criados para proteger os animais utilizados em pesquisa e ensino, como será demonstrado posteriormente. Segundo a professora de Técnica Cirúrgica da (FMVZ/USP), Julia Maria Matera, com a utilização da solução de Larssen modificada o número de animais sacrificados para aulas de práticas cirúrgicas passou de 440 cães saudáveis para 40 cães que já se encontravam mortos e foram preservados com a solução de Larssen.

\subsection{Vídeos Demonstrativos como método alternativo}

O segundo método alternativo para o ensino da teoria das práticas cirúrgicas é o que utiliza vídeos demonstrativos. Esses vídeos têm o benefício de terem baixo custo, considerando que o procedimento é feito uma única vez e gravado, o que possibilita a visualização do procedimento inúmeras vezes, inclusive em câmera lenta. No entanto, este método não é capaz de passar as dimensões sensíveis como volume, textura e dentre outras que só são possíveis pela experiência em reais dimensões.

A utilização de vídeos se torna muito eficaz se utilizada em conjunto com outros métodos de ensino, pois as aulas por vídeos seriam usadas na formação do conhecimento teórico, e para preencher as carências da formação da prática da técnica cirúrgica, haveria plantões e estágios em hospitais veterinários ou clínicas, assim como mutirões de castração para cães e gatos, feitos pelos alunos e acompanhados pelo professor responsável pela disciplina. Essa alternativa, além de formar o aluno para o conhecimento teórico, o formaria para as habilidades práticas, estando de acordo com o princípio ético da comparação da eficiência com a necessidade, pois teria a mesma eficiência da metodologia de aula convencional que utiliza animais mantidos em um biotério e são posteriormente sacrificados. 
FREITAS, A.I.A. e ROSATO, G.R. Estudo ético e científico sobre o uso de métodos alternativos no ensino de práticas cirúrgicas na Medicina Veterinária. PUBVET, Londrina, V. 5, N. 9, Ed. 156, Art. 1053, 2011.

Infelizmente, o uso de animais para o ensino é uma prática antiga, mas a possibilidade de se gravar as aulas em recursos áudio visuais já existe há mais de vinte anos. Em algumas faculdades em que os procedimentos são feitos apenas com intuitos demonstrativos, há muitos que ainda não são gravados, gerando o uso de mais animais vivos para cada nova demonstração, enquanto que o sacrifício de suas vidas poderia ser evitado pela possibilidade do uso de tal método.

\subsection{Programas de Computadores como métodos alternativos}

O terceiro método alternativo escolhido para o estudo de sua eficiência foram os programas de computadores especializados no treinamento de técnicas cirúrgicas. Em um estudo feito por $\mathrm{Smith}^{4}$ e alguns colaboradores, existem três categorias de modelos computadorizados: os softwares, que são representados por gráficos, sons e animações; as ferramentas didáticas integradas, em que os programas são combinados a sistemas audiovisuais; e o modelo mais avançado entre os três, que é a utilização de realidade virtual, em que os procedimentos cirúrgicos podem ser treinados em terceira dimensão, através de óculos especiais, que podem estar ligados a sistemas que refletem sensibilidade. ${ }^{5}$

Os programas de computadores que simulam uma realidade em terceira dimensão têm diversas vantagens, pois podem ser utilizados para o treinamento de diversos procedimentos cirúrgicos, desenvolvendo as habilidades e coordenações necessárias ao cirurgião sem colocar em risco nenhuma vida, o que cria a possibilidade de repetição do procedimento inúmeras vezes. Como desvantagem, podemos apontar o alto custo financeiro para aquisição de um programa de realidade virtual com sistemas que refletem

\footnotetext{
${ }^{4}$ SMITH, A; FOSSE, R; DEWHURST, D; SMITH, K. Educational Simulation models in the biomedical sciencies. ILARJ. 1997; 32(8): 82-88.

${ }^{5}$ DINIZ, R; DUARTE, A. L. A; OLIVEIRA, C. A. S; ROMITI, M. Animais em aulas práticas: podemos substituílos com a mesma qualidade de ensino?. Disponível em $<$ http://www.scielo.br/pdf/rbem/v30n2/v30n2a05.pdf $>$ Acesso em 18. Jul. 2010.
} 
FREITAS, A.I.A. e ROSATO, G.R. Estudo ético e científico sobre o uso de métodos alternativos no ensino de práticas cirúrgicas na Medicina Veterinária. PUBVET, Londrina, V. 5, N. 9, Ed. 156, Art. 1053, 2011.

sensibilidade. No entanto, esses programas podem demonstrar serem mais econômicos a longo prazo, se comparados a manutenção de um biotério.

\section{As vantagens do uso de métodos alternativos}

A utilização de novas tecnologias para formulação de uma nova metodologia de ensino de práticas cirúrgicas, observando os três métodos alternativos, que visam a substituição de animais vivos em comparação com a metodologia convencional, apresentam algumas vantagens e desvantagens em comum.

Como vantagem que se pretende obter com a utilização de métodos alternativos, que são comuns aos três métodos escolhidos para estudo deste trabalho, tem como o mais importante objetivo a formação de uma consciência ética no aluno que ao aprender a respeitar o valor da vida de um animal, se tornará um profissional com sensibilidade, sem desvincular o animal do valor de sua vida, o tratando como algo mais valioso que um simples objeto de estudo.

Outra vantagem em comum aos três métodos apresentados é a possibilidade de repetição do procedimento inúmeras vezes, sem colocar em risco e nem causar danos a nenhum animal, o que torna os métodos alternativos mais indicados didaticamente para a formação do próprio médico veterinário.

\section{Avaliação da ética normativa sobre o uso de métodos alternativos}

O estudo da ética normativa pode esclarecer as questões sobre as condições impostas à utilização de animais para fins didáticos, podendo ser também um reforço para a argumentação favorável à utilização de métodos alternativos no ensino de práticas cirúrgicas.

Para um melhor estudo da ética normativa, primeiramente se faz necessário considerar as regras e leis vigentes no Brasil, para isso será feito uma elaboração sobre a situação da utilização de animais vivos para fins 
FREITAS, A.I.A. e ROSATO, G.R. Estudo ético e científico sobre o uso de métodos alternativos no ensino de práticas cirúrgicas na Medicina Veterinária. PUBVET, Londrina, V. 5, N. 9, Ed. 156, Art. 1053, 2011.

didáticos, considerando a possibilidade da substituição por métodos alternativos.

Uma das mais importantes regras e princípios humanitários para utilização de animais em pesquisa e ensino foi formulada em 1959, por dois ingleses, Russel e Burch. Essa regra é chamada de "lei dos três erres (3R)", no inglês, replacemente, reduction e refinement. O primeiro " $R$ ", replacemente, pode ser traduzido por substituição, ou seja, substituir o uso de animais, quando possível, por outros métodos; o segundo "R", reduction, se traduz por redução, utilizar o menor número possível de animais para obtenção do resultado desejado; e o terceiro " $\mathrm{R}$ ", refinement, que se traduz por refinamento, é entendido como o refinamento das técnicas de ensino e pesquisa, com fins de evitar danos desnecessários aos animais envolvidos. ${ }^{6}$

\subsection{A lei de crimes ambientais}

A lei de crimes ambientais condena a prática do uso de animais perante a possibilidade de substituição por métodos alternativos. De acordo com a Lei 9.605, de 12 de fevereiro de 1998, sofrerá pena de três meses a um ano, para atos como os que podemos observar no Art. 32 que qualquer prática que cause danos a animais silvestres domésticos ou domesticados, nativos ou exóticos, e no $\S 1^{\circ}$ as mesmas penas são aplicadas a quem realizar experiências dolorosas ou cruéis em animais vivos, ainda que para fins didáticos ou científicos, quando existirem recursos alternativos. ${ }^{7}$

A lei de crimes ambientais, como se pode observar, torna o uso de animais vivos para fins didáticos um ato ilegal mediante a possibilidade de métodos alternativos. Muitos pesquisadores que ainda utilizam os métodos convencionais, para tentar justificar sua metodologia perante a existência da lei tentam argumentar que o uso de métodos alternativos não consegue suprir

6 BONELLA, A. E. Animais em laboratório e lei Arouca. Disponível em $<$ http://www.scielo.br/scielo.php?script=sci_arttext\&pid=S1678-31662009000300008\&lng=en\&nrm=iso\&tlng=pt $>$ Acesso em 15 . Jan. 2010.

${ }^{7}$ TUDURY, E. A; POTIER, G. M. A. Métodos substitutivos ao uso de animais vivos no ensino. Disponível em <http://www.bio.ufpr.br/ceea/doc/artigos/AlternativasensinoTudury92-95.pdf > Acesso em 18. Jul. 2010 
FREITAS, A.I.A. e ROSATO, G.R. Estudo ético e científico sobre o uso de métodos alternativos no ensino de práticas cirúrgicas na Medicina Veterinária. PUBVET, Londrina, V. 5, N. 9, Ed. 156, Art. 1053, 2011.

as necessidades exigidas ao ensino de práticas cirúrgicas. Como já foi demonstrado, este argumento se torna inválido perante a demonstração da eficiência dos métodos alternativos e a metodologia ideal para suprir suas possíveis carências.

\subsection{A resolução do Conselho Federal de Medicina Veterinária}

Todo médico veterinário deve seguir um conjunto de normas que estão nas resoluções do Conselho Federal de Medicina Veterinária, isso implica que o corpo docente e as aulas de práticas cirúrgicas estão submetidos a essas normas. Os métodos alternativos são metodologias humanitárias de ensino. $\mathrm{Na}$ Resolução n722, de 16 de agosto de 2002, no capítulo I sobre os princípios e fundamentos que o Médico Veterinário deve seguir, o Art. $4^{\circ}$ diz que no exercício profissional, é preciso o médico usar procedimentos humanitários para evitar sofrimento e dor ao animal. ${ }^{8}$ Podemos interpretar que para o docente responsável pelo ensino de práticas cirúrgicas, sendo esse o seu exercício profissional, é seu dever usar os métodos que não provoquem dor ou sofrimento aos animais, como é o caso dos métodos alternativos.

A resolução não se omite sobre o uso de métodos alternativos, podendo ser observado no capítulo II, Art. $6^{\circ}$, que trata dos deveres do médico veterinário, que é sempre preciso aprimorar os conhecimentos e usar o melhor do progresso científico em benefício dos animais e dos homens. ${ }^{9}$ Neste artigo, o uso de métodos alternativos, que existem tanto para o beneficio dos animais não-humanos quanto para os alunos humanos, pode ser visto como um dever do docente em busca do seu conhecimento e devem ser utilizados não somente nas práticas cirúrgicas, mas em todas as práticas didáticas e científicas que causem danos aos animais.

\footnotetext{
${ }^{8}$ CONSELHO REGIONAL DE MEDICINA VETERINÁRIA. Resolução ${ }^{\circ} 722$, de 16 de agosto de 2002. Regulamenta os princípios e fundamentos do médico veterinário. Lex: Ética profissional do médico veterinário, Belo Horizonte, MG, 16 ago. 2002

9 CONSELHO REGIONAL DE MEDICINA VETERINÁRIA. Resolução nº722, de 16 de agosto de 2002. Regulamenta os princípios e fundamentos do médico veterinário. Lex: Ética profissional do médico veterinário, Belo Horizonte, MG, 16 ago. 2002
} 
FREITAS, A.I.A. e ROSATO, G.R. Estudo ético e científico sobre o uso de métodos alternativos no ensino de práticas cirúrgicas na Medicina Veterinária. PUBVET, Londrina, V. 5, N. 9, Ed. 156, Art. 1053, 2011.

No capítulo X da Resolução, em seu Art. 25, o uso de animais, para aulas práticas e experimentação científica, deve ser feito somente em casos justificáveis, que possam resultar em beneficio da qualidade de ensino, da vida do animal e do homem, e apenas quando não houver alternativas cientificamente válidas. Neste artigo, considerando que os métodos alternativos foram devidamente comprovados como métodos científicos eficientes e que produzem vários benefícios didáticos, é dever do médico veterinário em sua relação com o animal não-humano, não utilizar os animais vivos para fins didáticos e científicos.

\subsection{A Constituição Federal}

Na Constituição da República Federativa do Brasil, podemos encontrar argumentos para comprovar que o uso de animais vivos para fins didáticos consiste em um ato reprovável por provocar crueldade contra os mesmos. É estipulado no artigo 225, $\S 10$, inciso VII, a submissão dos animais à crueldade, juntamente com os atos que provoquem extinção de suas espécies.

Os métodos convencionais utilizam animais vivos para o ensino de práticas cirúrgicas. Muitos desses animais não se encontram em estado moribundo e geralmente não precisam dos procedimentos aos quais são submetidos, sendo também eutanasiados ao final do treinamento. Considerando a possibilidade de substituição dos animais vivos por animais que já se encontravam mortos e foram perfeitamente conservados ou de animais que realmente precisem de um procedimento cirúrgico e de tratamento pós-operatório, o uso de animais vivos e saudáveis e sua posterior eutanásia se faz desnecessária e consiste em um ato de crueldade para com os animais sendo, portanto, reprovável e ilegal perante a ética e o direito.

\section{Conclusão}

1. O uso de animais vivos para o ensino de práticas cirúrgicas não pode ser justificado por argumentos éticos ou científicos. Há possibilidade de substituição dos métodos convencionais por métodos alternativos, na medida 
FREITAS, A.I.A. e ROSATO, G.R. Estudo ético e científico sobre o uso de métodos alternativos no ensino de práticas cirúrgicas na Medicina Veterinária. PUBVET, Londrina, V. 5, N. 9, Ed. 156, Art. 1053, 2011.

em que esses apresentem igual ou superior qualidade, se utilizados em conjunto.

2. Com o estudo da ética prática podemos concluir que o uso de animais vivos é um ato reprovável por não respeitar o valor da vida dos animais e seu interesse em continuar vivo sem sofrer danos.

3. Os métodos convencionais apresentam graves falhas e o uso exclusivo de animais vivos não possibilita o treinamento constante dos procedimentos cirúrgicos, sendo insuficientes para a formação de um cirurgião. Além disso, provoca em alguns alunos uma perda da sensibilidade a respeito do valor da vida de um animal.

4. Os métodos alternativos apresentam eficiência no ensino de práticas cirúrgicas, possibilitando um treinamento que cumpre com os objetivos esperados, além de respeitar os princípios que valorizem a vida do animal.

5. Perante a ética normativa, a Constituição Federal, a Resolução do Conselho Federal de Medicina Veterinária e a Lei de Crimes Ambientais, o uso de animais vivos para fins didáticos, considerando a possibilidade de substituição por métodos alternativos, se torna um ato ilegal, reprovável e passível de penas.

\section{Referências}

BONELLA, A. E. Animais em laboratório e lei Arouca. Disponível em $<$ http://www.scielo.br/scielo.php?script=sci arttext\&pid $=$ S1678-

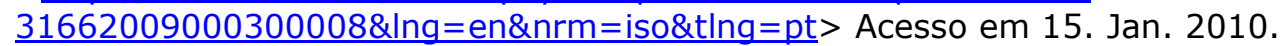

CONSELHO REGIONAL DE MEDICINA VETERINÁRIA. Resolução n722, de 16 de agosto de 2002. Regulamenta os princípios e fundamentos do médico veterinário. Lex: Ética profissional do médico veterinário, Belo Horizonte, MG, 16 ago. 2002.

CONSELHO REGIONAL DE MEDICINA VETERINÁRIA. Resolução $n^{\circ} 722$, de 16 de agosto de 2002. Regulamenta os princípios e fundamentos do médico veterinário. Lex: Ética profissional do médico veterinário, Belo Horizonte, MG, 16 ago. 2002.

DINIZ, R; DUARTE, A. L. A; OLIVEIRA, C. A. S; ROMITI, M. Animais em aulas práticas: podemos substituí-los com a mesma qualidade de ensino?. Disponível em <http://www.scielo.br/pdf/rbem/v30n2/v30n2a05.pdf> Acesso em 18. Jul. 2010.

SILVA, R. M. G; MATERA, J. M; MACIEL, A. A. C. Avaliação do método do ensino da técnica cirúrgica utilizando cadáveres quimicamente preservados. Disponível em <http://www.hostcentral.com.br/crmv/PDF/v6n1a10.pdf> Acesso em 20. Jul. 2010 
FREITAS, A.I.A. e ROSATO, G.R. Estudo ético e científico sobre o uso de métodos alternativos no ensino de práticas cirúrgicas na Medicina Veterinária. PUBVET, Londrina, V. 5, N. 9, Ed. 156, Art. 1053, 2011.

SINGER, Peter. Ética Prática. Tradução de Jefferson Luiz Camargo. São Paul: Martins Fontes, 2006. p 65. Tradução de: Practical Ethics.

SMITH, A; FOSSE, R; DEWHURST, D; SMITH, K. Educational Simulation models in the biomedical sciencies. ILARJ. 1997; 32(8): 82-88.

TUDURY, E. A; POTIER, G. M. A. Métodos substitutivos ao uso de animais vivos no ensino. Disponível em <http://www.bio.ufpr.br/ceea/doc/artigos/AlternativasensinoTudury9295.pdf > Acesso em 18. Jul. 2010. 